\title{
CodonGenie: optimised ambiguous codon design tools (\#16109)
}

First revision

Please read the Important notes below, the Review guidance on page 2 and our Standout reviewing tips on page 3 . When ready submit online. The manuscript starts on page 4.

Important notes

\section{Editor}

James Procter

Files

1 Tracked changes manuscript(s)

1 Rebuttal letter(s)

1 Figure file(s)

1 Table file(s)

Please visit the overview page to download and review the files not included in this review PDF.

Declarations

No notable declarations are present 
Please read in full before you begin

\section{How to review}

When ready submit your review online. The review form is divided into 5 sections. Please consider these when composing your review:

\section{BASIC REPORTING}

2. EXPERIMENTAL DESIGN

3. VALIDITY OF THE FINDINGS

4. General comments

5. Confidential notes to the editor

You can also annotate this PDF and upload it as part of your review

To finish, enter your editorial recommendation (accept, revise or reject) and submit.

\section{BASIC REPORTING}

Clear, unambiguous, professional English language used throughout.

Intro \& background to show context. Literature well referenced $\&$ relevant.

Structure conforms to PeerJ standards, discipline norm, or improved for clarity.

Figures are relevant, high quality, well labelled \& described.

Raw data supplied (see PeerJ policy).

\section{EXPERIMENTAL DESIGN}

Original primary research within Scope of the journal.

Research question well defined, relevant $\&$ meaningful. It is stated how the research fills an identified knowledge gap.

Rigorous investigation performed to a high technical \& ethical standard.

Methods described with sufficient detail \& information to replicate.

\section{VALIDITY OF THE FINDINGS}

Impact and novelty not assessed. Negative/inconclusive results accepted. Meaningful replication encouraged where rationale $\&$ benefit to literature is clearly stated.

Data is robust, statistically sound, $\&$ controlled.
Conclusions are well stated, linked to original research question \& limited to supporting results.

Speculation is welcome, but should be identified as such.

The above is the editorial criteria summary. To view in full visit https://peerj.com/about/editorialcriteria/ 
The best reviewers use these techniques

Tip

\author{
Support criticisms with \\ evidence from the text or from \\ other sources
}

\section{Give specific suggestions on how to improve the manuscript}

\section{Comment on language and grammar issues}

Organize by importance of the issues, and number your points

\section{Example}

Smith et al (J of Methodology, 2005, V3, pp 123) have shown that the analysis you use in Lines 241-250 is not the most appropriate for this situation. Please explain why you used this method.

Your introduction needs more detail. I suggest that you improve the description at lines 57- 86 to provide more justification for your study (specifically, you should expand upon the knowledge gap being filled).

The English language should be improved to ensure that your international audience can clearly understand your text. I suggest that you have a native English speaking colleague review your manuscript. Some examples where the language could be improved include lines 23, 77, 121, 128 - the current phrasing makes comprehension difficult.

1. Your most important issue

2. The next most important item

3. ...

4. The least important points

Line 56: Note that experimental data on sprawling animals needs to be updated. Line 66: Please consider exchanging "modern" with "cursorial".

I thank you for providing the raw data, however your supplemental files need more descriptive metadata identifiers to be useful to future readers. Although your results are compelling, the data analysis should be improved in the following ways: $A A, B B, C C$

I commend the authors for their extensive data set, compiled over many years of detailed fieldwork. In addition, the manuscript is clearly written in professional, unambiguous language. If there is a weakness, it is in the statistical analysis (as I have noted above) which should be improved upon before Acceptance.
Comment on strengths (as well as weaknesses) of the manuscript




\title{
CodonGenie: optimised ambiguous codon design tools
}

\author{
Neil Swainston ${ }^{\text {Corresp., }}{ }^{\text {， Andrew Currin }}{ }^{1}$ ， Lucy Green ${ }^{1}$ ， Rainer Breitling ${ }^{1,2}$ ， Philip J Day ${ }^{3}$, Douglas B Kell ${ }^{1,2}$ \\ 1 Manchester Centre for Synthetic Biology of Fine and Speciality Chemicals (SYNBIOCHEM), University of Manchester, Manchester, United Kingdom \\ 2 School of Chemistry, University of Manchester, Manchester, United Kingdom \\ 3 Faculty of Biology, Medicine and Health, University of Manchester, Manchester, United Kingdom \\ Corresponding Author: Neil Swainston \\ Email address: neil.swainston@manchester.ac.uk
}

CodonGenie, freely available from http://codon.synbiochem.co.uk, is a simple web application for designing ambiguous codons to support protein mutagenesis applications. Ambiguous codons are derived from specific heterogeneous nucleotide mixtures, which create sequence degeneracy when synthesised in a DNA library. In directed evolution studies, such codons are carefully selected to encode multiple amino acids. For example, the codon NTN, where the code $\mathrm{N}$ denotes a mixture of all four nucleotides, will encode a mixture of phenylalanine, leucine, isoleucine, methionine and valine. Given a user-defined target collection of amino acids matched to an intended host organism, CodonGenie designs and analyses all ambiguous codons that encode the required amino acids. The codons are ranked according to their efficiency in encoding the required amino acids while minimising the inclusion of additional amino acids and stop codons. Organism-specific codon usage is also considered. 


\section{CodonGenie: optimised ambiguous codon design tools}

2 1, Neil Swainston, ${ }^{1}$ Andrew Currin, ${ }^{1}$ Lucy Green, ${ }^{1,2}$ Rainer Breitling, ${ }^{3}$ Philip J Day, ${ }^{1,2}$ Douglas B 3 Kell

$4{ }^{1}$ Manchester Centre for Synthetic Biology of Fine and Speciality Chemicals (SYNBIOCHEM), 5 Manchester Institute of Biotechnology, University of Manchester, Manchester M1 7DN, United 6 Kingdom.

$7 \quad{ }^{2}$ School of Chemistry, University of Manchester, Manchester M13 9PL, United Kingdom.

$8{ }^{3}$ Faculty of Biology, Medicine and Health, University of Manchester, Manchester M13 9PL, 9 United Kingdom.

$10 *$ Corresponding author.

11 Abstract

12 CodonGenie, freely available from http://codon.synbiochem.co.uk, is a simple web application

13 for designing ambiguous codons to support protein mutagenesis applications. Ambiguous codons

14 are derived from specific heterogeneous nucleotide mixtures, which create sequence degeneracy when synthesised in a DNA library. In directed evolution studies, such codons are carefully selected to encode multiple amino acids. For example, the codon NTN, where the code N denotes a mixture of all four nucleotides, will encode a mixture of phenylalanine, leucine, isoleucine, methionine and valine. Given a user-defined target collection of amino acids matched to an intended host organism, CodonGenie designs and analyses all ambiguous codons that encode the required amino acids. The codons are ranked according to their efficiency in encoding the required amino acids while minimising the inclusion of additional amino acids and stop codons. Organism-specific codon usage is also considered.

\section{Introduction}

Site-directed mutagenesis of DNA is an established technique of generating libraries of DNA variants in a controlled manner, and has applications in a range of fields, primarily that of protein engineering (Jäckel, Kast \& Hilvert, 2008), but also in more fundamental research including the study of sequence-to-fitness relationships (Hietpas et al., 2011). The design of mutant protein libraries typically involves a manual process in which required sites for mutation are selected and ambiguous codons (those containing mixtures of nucleotides) designed to introduce controlled variation in these positions.

In this process, one may wish to design a codon to specify any subset of amino acids in a given position. Since each amino acid may be included in the subset or otherwise, the number of possible subsets is $2^{20}-1$, i.e. there are 1,048,575 possible subsets of 20 amino acids. (Each of 
34 the sets can be represented by a 20-digit binary number, where a one at position $n$ indicates that amino acid $n$ is included in the set, and a zero indicates that it is absent. There are $2^{20}$ such numbers, but one of them represents the empty set and is thus not counted here.) Not all of these 1,048,575 subsets of 20 amino acids are uniquely designable using ambiguous codons, of which there are only 3375. (There are $15\left(=2^{4}-1\right)$ relevant nucleotide codes ("letters"), ranging from the completely unambiguous $A, C, G$ and $T$ representing a single nucleotide, to the completely ambiguous $\mathrm{N}$ representing all 4 nucleotides (Cornish-Bowden, 1985). There are $15^{3}=3375$ triplet codons that can be assembled from this 15-letter alphabet of ambiguous codes, compared to the $4^{3}=64$ codons that can be constructed from the standard 4-letter alphabet of unambiguous nucleotides.)

Given the degeneracy of the codon table, there are often multiple ways to encode a chosen set of amino acids. The experimenter must a) decide if it is feasible to encode all desired amino acids (Mena \& Daugherty, 2005); b) determine whether this creates an acceptable number of sequence combinations (depending on screening capability and throughput) (Currin et al., 2015; Kille et al., 2013; Lutz, 2010; Pines et al., 2015); and c) consider the codon usage of the organism to be used (Nakamura, Gojobori \& Ikemura, 2000). It therefore follows that the design of ambiguous codons is non-trivial.

CodonGenie is therefore introduced to provide a quick and easy-to-use means of designing optimal ambiguous codons, considering the above parameters according to the user input, and ranking the ambiguous codons with respect to their suitability for expression in a target host organism. The tool is designed to be both human- and computer-readable, providing both a simple web browser interface and a RESTful webservice API.

\section{Materials \& Methods}

Algorithm

The standard codon table is such that 17 of the 20 naturally occurring amino acids are encoded by codons with fixed bases in the first and second positions, with the third "wobble"-position allowing variation that accounts for the degeneracy of the DNA code. Determining optimal ambiguous codons for combinations of amino acids involves the following process, which is optimized for computational efficiency, compared to a brute-force examination of all possible ambiguous codons:

Align the first two positions and select the most specific ambiguous bases to encode the alignment. For example, with the combination asparagine and isoleucine (encoded by AA [CT] and $A T[A C T]$ respectively), the alignment of the first two positions is $A[A T]$, i.e. $A W$.

67 All combinations of aligned wobble positions are calculated, i.e. $[\mathrm{CA}],[\mathrm{CC}],[\mathrm{CT}],[\mathrm{TA}]$, $68[\mathrm{TC}],[\mathrm{TT}]$. These are then collapsed into unique sets, in this example giving $[\mathrm{CA}], \mathrm{C},[\mathrm{CT}]$, 
$69[\mathrm{TA}]$ and $\mathrm{T}$.

70 The first two and wobble position bases are combined to produce candidate ambiguous codons,

71 which are scored as described below.

72 Three amino acids (leucine, arginine and serine) cannot be simply encoded by codons with fixed 73 bases in the first and second positions. (For example, both CTN and TT [AG] encode leucine.)

74 For combinations including these more complex residues, the above algorithm is performed for 75 each encoding and the results combined.

76 Note that CodonGenie returns not only the most "specific" ambiguous codons, that is, the codons 77 that provide the fewest DNA variants whilst encoding all target amino acids. Providing results 78 that include less specific ambiguous codons, which may also encode additional amino acids, 79 allows the user to perform a trade-off between library size and codon specificity, depending on 80 the experimental objective. A smaller library is generally advantageous for screening purposes, 81 but may contain codons that are unfavoured by the target host organism.

\section{Scoring}

83 The goal of the scoring scheme is to preferentially rank the most efficient ambiguous codons.

84 That is, the ambiguous codons that encodes all of the required amino acids while minimising the 85 encoding on non-desired amino acids.

86 The score for an ambiguous codon is therefore defined as the mean of the value, $v_{i}$, of each of the 87 codons that it encodes. For codons that encode required amino acids, $v_{i}$ is the ratio of the 88 frequency of the codon $\mathrm{f}_{\mathrm{i}}$ and the frequency of the most frequent synonymous codon $f_{j}$ for the 89 amino acid that it encodes. For codons that encode non-required amino acids, $v_{i}$ is zero.

$$
\text { score }=\frac{1}{|C|} \sum_{i \in C} v_{i} \quad, \text { where }
$$

$$
v_{i}=\left\{\begin{array}{cc}
\frac{f_{i}}{\max \left(\left\{f_{j}: j \in S_{i}\right\}\right)} & i \in R \\
0 & i \notin R
\end{array}\right.
$$

$C=\{$ all variants of ambiguous codon $c\}$

$A=\{$ target amino acids $\}$

$a_{i}$ : amino acid encoded by codon $i \in C$

$f_{i}$ : codon usage frequency of codon $i \in C$

$$
S_{i}=\left\{j: a_{j}=a_{i}\right\} \quad \text { Set of synonymous codons of codon } i
$$

$R=\left\{i \in C: a_{i} \in A\right\}$ Set of codon variants of $c$ encoding target amino acids 
98 This scoring algorithm thus achieves a principled trade-off between codon specificity, library

99 size and codon favourability (according to the codon usage preferences of the target organism).

\section{Web service access}

101 CodonGenie also offers a RESTful web service interface, supporting its integration with

102 software pipelines. The Design method can be accessed by specifying required amino acids and

103 required host organism (as an NCBI Taxonomy id (Federhen, 2012)) as follows:

104 http://codon.synbiochem.co.uk/codons?aminoAcids=DE\&organism=4932

105 Similarly, the Analyse method can be accessed by specifying a variant codon and the required 106 organism:

\section{7 http://codon.synbiochem.co.uk/codons?codon=NSS\&organism=4932}

108 CodonGenie also provides web service interfaces for accessing supported organisms. The first 109 allows all organisms to be listed, showing NCBI Taxonomy id and name, and the second allows

110 the collection to be searched according to a given term:

111 http://codon.synbiochem.co.uk/organisms/

112 http://codon.synbiochem.co.uk/organisms/escher

113 In all cases, results are returned in json format.

\section{Distribution}

115 The web application is freely available from http://codon.synbiochem.co.uk. CodonGenie is

116 written in Python (using the Flask framework) and HTML / Javascript (using the Bootstrap and 117 AngularJS libraries) and is packaged as a Docker application for ease of deployment. Source

118 code is available from https://github.com/synbiochem/CodonGenie.

\section{Results and Discussion}

120 CodonGenie provides a simple web interface affording two functions: a) the design, and b) the 121 analysis of ambiguous codons. Considering the Design module, the user specifies the 122 combination of amino acids to be encoded and an organism in which the library will be 123 expressed. The codon usage table is automatically extracted from the Codon Usage Database

124 (Nakamura, Gojobori \& Ikemura, 2000), which as of May 2017 provided support for 35,792 125 organisms. CodonGenie then calculates suitable ambiguous codons and presents these in an 126 interactive table (see Figure 1).

127 The Analyse module provides the functionality of checking an existing ambiguous codon. Users 128 specify a variant codon and required host organism, and the results returned indicate which 129 amino acids are encoded along with their codon usage frequency. 
130 The benefit of CodonGenie can be exemplified by the design of an ambiguous codon to encode

131 non-polar amino acids phenylalanine, leucine, isoleucine, methionine and valine. A simple and

132 widely used ambiguous codon to encode this subset is NTN, which equates to 16 DNA variants.

133 However, CodonGenie identifies that these same amino acids can be encoded by the DTK codon

134 (where D denotes [AGT] and K denotes [GT] ) using 6 variants. Selecting DTK therefore means

135 fewer enzyme variants need to be screened to test all sequence combinations. This benefit is

136 particularly significant when encoding multiple variant codons. For example, when using 3 DTK

137 codons the library size is reduced from $4096\left(16^{3}\right)$ to $213\left(6^{3}\right)$ combinations.

138 An example of the importance of considering codon usage of the target host organism can be

139 seen when considering the design of an ambiguous codon to encode the set of five non-polar

140 amino acids ( F, I, L, M and V) considered above. For E. coli, the preferred codon is DTK

141 (ATG $|\mathrm{T}| \mathrm{GT}$ ), with a score of 0.88 . DTS (ATG $|\mathrm{T}| \mathrm{GC}$ ) also encodes all five amino acids using 6

142 variants, but with a score of 0.68. In Streptomyces coelicolor - a commonly used host for

143 antibiotic production (Pickens et al., 2011), the ranking is reversed, with DTS being preferred

144 with a score of 0.79 , substantially higher than that of 0.29 for DTK. The reason for this can be

145 found in the codon usage frequencies of each of these organisms, as shown in Table 1: The

146 codons DTK and DTS differ by specifying either GT or GC in the third position, respectively.

147 Taking the example of encoding phenylalanine, F, the codon TTT encoded by ambiguous codon

148 DTK is preferred over TTC (encoded by DTS) in E. coli by a frequency of 0.64 to 0.36 . By

149 contrast, $S$. coelicolor strongly prefers TTC to TTT to encode F, with frequencies of 0.97 to 0.03 ,

150 respectively. A similar preference is observable in the codon usage frequencies for encoding

151 isoleucine, I, in $S$. coelicolor, where ATC has a frequency of 0.95 compared to that of 0.03 for

152 ATT. Thus, $S$. coelicolor has a strong preference for the variant codon containing $C$ in the

153 "wobble" position, and this is reflected in the scores of 0.79 for DTS and 0.29 for DTK.

154 Organism-specific codon usage is therefore a key consideration in the design of ambiguous

155 codons for a given host.

156 CodonGenie adds to a toolkit of existing software tools for ambiguous codon selection, which

157 includes AA-Calculator (Firth \& Patrick, 2008) and DYNAMCC (Halweg-Edwards et al., 2016).

158 In contrast to AA-Calculator, CodonGenie ranks designed ambiguous codon based on their

159 suitability for use in a given host organism. DYNAMCC also scores designed codons but offers

160 complementary functionality to CodonGenie, as it designs sets of ambiguous codons to encode a

161 set of amino acids with no off-target amino acid encoding and minimal redundancy. CodonGenie

162 designs single ambiguous codons to encode a desired set of amino acids, which may also include

163 off-target amino acids, allowing users to make a conscious trade-off between a larger library and

164 the ease of generating such a library with a single ambiguous codon.

165 The above example of Table 1 illustrates a key difference between CodonGenie and

166 DYNAMCC. Where CodonGenie will provide a list of individual ambiguous codons that will

167 encode all desired amino acids (and potentially additional, off-target amino acids), DYNAMCC 
168 returns a single, best-scoring set of ambiguous codons that encode all desired amino acids with

169

170

171

172

173

174

175

176

177

178

179

180

181

182

183

184

185

186

187

188

189

190

191

192

193

194

195

196

197

198

199

200

201

202

203

204

205

minimal redundancy. In the case of F, I, L, M and V, DYNAMCC returns the set of codons WTT (encoding $\mathrm{F}$ and I and L) and VTG (encoding M and V). The advantage of the DYNAMCC approach is in increased efficiency of the library: five DNA variants encode the five desired amino acids, while CodonGenie's solution of DTK or DTS encode six DNA variants, thus producing a larger library. The advantage of CodonGenie's solution lies in the ease in which the library can be produced with a single ambiguous codon.

CodonGenie provides a clean, intuitive web-based user interface which requires minimal user input, and which takes advantage of modern web-application development libraries such as AngularJS and Bootstrap. AngularJS (https://angularjs.org), developed and maintained by Google, provides a framework for the rapid development of modular, testable single-page web applications. Bootstrap (http://getbootstrap.com), initially developed at Twitter, provides a library of reusable user interface "widgets", such as forms, auto-fill boxes, tables, etc. Using freely available yet commercially developed libraries such as these confers a number of advantages: From a development perspective, the libraries are easy to use, are well documented and are thoroughly tested on a range of browsers (including those on mobile phones and tablets) being used perhaps billions of times a day worldwide. More importantly, the user experience is improved through use of well-developed modules that in many cases users have experienced numerous times previously in various other web applications. As a result, CodonGenie can provide a simple, easy-to-use interface that requires no documentation and can run on many platforms with the minimum of development effort.

CodonGenie is designed to follow the concept of "microservices" (Williams et al., 2016). Microservice architecture advocates the breaking down of large, monolithic applications into simple, atomic services of limited scope of functionality. By deconstructing large applications or pipelines (such as a DNA design tool) into a collection of independent units (such as a codon design module), the individual microservices can be developed, tested and deployed in isolation, increasing their reliability and reusability. CodonGenie follows this paradigm (the entire application consists of $\sim 700$ lines of code) and allows for integration into larger applications by providing a simple computer-readable RESTful web service API, as well as making itself available as a Docker container (Belmann et al, 2015; Leprevost et al., 2017), allowing users to easily redeploy their own instantiation on individual computers and services, or cloud-based platforms.

One example of the use of the CodonGenie as a microservice within a larger application is in automating the design of a synthetic DNA sequence to encode a protein sequence generated from a multiple sequence alignment. Consider a multiple sequence alignment of a hypothetic active site of an enzyme: 
206

207

208

209

210

211

212

213

214

215

216

217

218

219

220

221

222

223

224

225

226

227

228

229

230

231

232

233

234

235

236

237

238

239
PLHLR

PMNMR

PVHMR

The CodonGenie webservice facilitates the writing of a simple script to automate the process of designing a synthetic DNA sequence that captures the variation encoded in this alignment. By iterating through the alignment, the set of amino acids required at each position can be collected ( $\{P\}$ for position 1, $\{F I L M V\}$ for position 2, etc.). These sets can be submitted to the CodonGenie webservice (along with a desired host organism) and a synthetic DNA sequence built up from the highest-scoring ambiguous codon returned. In practice, CodonGenie would produce the following DNA sequence for E. coli:

CCG | DTK | VMT | MTG | CGT

In this example, the first codon (CCG) is not strictly an ambiguous codon, as it contains no ambiguous nucleotides, given that a single amino acid, $\mathrm{P}$, is required in the first position. The codon returned is the therefore the most frequent codon for encoding proline in E. coli. The second codon is the optimum codon for encoding F, I, L, M and V, as shown previously.

This example shows the benefit of offering webservice access to the CodonGenie method. While manually designing an optimised DNA sequence for a short alignment such as this is tractable, performing a similar operation on a longer alignment or a number of alignments in a manual fashion would not be feasible. Example code performing this simple operation is available (https://github.com/synbiochem/CodonGenie/blob/master/codon_genie/example/align.py), giving an indication of the ease with which CodonGenie could be incorporated into more comprehensive DNA design pipelines.

\section{Conclusion}

CodonGenie provides two simple-to-use yet valuable tools that aid the design of variant protein libraries in mutagenesis and directed evolution studies. Through both its web and web service interfaces, CodonGenie is amenable to future integration with new and existing variant library design software tools (Swainston et al, 2014). Its modular and open-source format allows for straightforward adaptation to emerging needs in the synthetic biology community, in particular the consideration of augmented genetic codes and expanded genetic alphabets (Lajoie et al, 2013; Zhang, 2017).

\section{References}

Belmann, P., Dröge, J., Bremges, A., McHardy, A.C., Sczyrba, A., Barton, M.D. (2015) Bioboxes: standardised containers for interchangeable bioinformatics software. Gigascience. 4 : 47. 
240 Cornish-Bowden A. (1985) Nomenclature for incompletely specified bases in nucleic acid

241 sequences: recommendations 1984. Nucleic Acids Res. 13: 3021-30.

242 Currin, A., Swainston, N., Day, P. J., and Kell, D. B. (2015) Synthetic biology for the directed

243 evolution of protein biocatalysts: navigating sequence space intelligently. Chem Soc Rev. 44:

$244 \quad 1172-239$.

245 Federhen, S. (2012) The NCBI Taxonomy database. Nucleic Acids Res. 40: D136-43.

246 Firth AE, Patrick WM. (2008) GLUE-IT and PEDEL-AA: new programmes for analyzing

247 protein diversity in randomized libraries. Nucleic Acids Res. 36: W281-5.

248 Halweg-Edwards AL, Pines G, Winkler JD, Pines A, Gill RT. (2016) A Web Interface for Codon

249 Compression. ACS Synth Biol. 5: 1021-3.

250 Hietpas, R.T., Jensen, J.D., Bolon, D.N. (2011) Experimental illumination of a fitness landscape.

251 Proc Natl Acad Sci U S A. 108: 7896-901.

252 Jäckel, C., Kast, P., Hilvert, D. (2008) Protein design by directed evolution. Annu Rev Biophys.

253 37: 153-73.

254 Kille, S., Acevedo-Rocha, C.G., Parra, L.P., Zhang, Z.G., Opperman, D.J., Reetz, M.T.,

255 Acevedo, J.P. (2013) Reducing codon redundancy and screening effort of combinatorial protein

256 libraries created by saturation mutagenesis. ACS Synth Biol. 2: 83-92.

257 Lajoie, M.J., Rovner, A.J., Goodman, D.B., Aerni, H.R., Haimovich, A.D., Kuznetsov, G.,

258 Mercer, J.A., Wang, H.H., Carr, P.A., Mosberg, J.A., Rohland, N., Schultz, P.G., Jacobson J,M.,

259 Rinehart, J., Church, G.M., Isaacs, F.J. (2013) Genomically recoded organisms expand

260 biological functions. Science. 342: 357-60.

261 Leprevost, F.D., Grüning, B.A., Alves Aflitos, S., Röst, H.L., Uszkoreit, J., Barsnes, H., Vaudel,

262 M., Moreno, P., Gatto, L., Weber, J., Bai, M., Jimenez, R.C., Sachsenberg, T., Pfeuffer, J., Vera

263 Alvarez, R., Griss, J., Nesvizhskii, A.I., Perez-Riverol, Y. (2017) BioContainers: An open-source

264 and community-driven framework for software standardization. Bioinformatics. doi:

265 10.1093/bioinformatics/btx192. [Epub ahead of print]

266 Lutz, S. (2010) Beyond directed evolution--semi-rational protein engineering and design. Curr

267 Opin Biotechnol. 21: 734-43.

268 Mena, M.A., Daugherty, P.S. (2005) Automated design of degenerate codon libraries. Protein

269 Eng Des Sel. 18: 559-61.

270 Mercer, J.A., Wang, H.H., Carr, P.A., Mosberg, J.A., Rohland, N., Schultz, P.G., Jacobson, J.M.,

271 Rinehart, J., Church, G.M., Isaacs, F.J. (2013) Genomically recoded organisms expand

272 biological functions. Science. 342: 357-60. 
273 Nakamura, Y., Gojobori, T., and Ikemura, T. (2000) Codon usage tabulated from the

274 international DNA sequence databases: status for the year 2000. Nucl Acids Res. 28: 292.

275 Pickens, L.B., Tang, Y., and Chooi, Y.H. (2011) Metabolic engineering for the production of 276 natural products. Annu Rev Chem Biomol Eng. 2: 211-36.

277 Pines G, Pines A, Garst AD, Zeitoun RI, Lynch SA, Gill RT. (2015) Codon compression

278 algorithms for saturation mutagenesis. ACS Synth Biol. 4: 604-14.

279 Swainston, N., Currin, A., Day, P. J., and Kell, D. B. (2014) GeneGenie: optimized oligomer 280 design for directed evolution. Nucleic Acids Res. 42: W395-400.

281 Williams, C.L., Sica, J.C., Killen, R.T., Balis, U.G. (2016) The growing need for microservices 282 in bioinformatics. J Pathol Inform. 7: 45.

283 Zhang, Y., Lamb, B.M., Feldman, A.W., Zhou, A.X., Lavergne, T., Li, L., Romesberg, F.E. 284 (2017) A semisynthetic organism engineered for the stable expansion of the genetic alphabet. 285 Proc Natl Acad Sci U S A. 114: 1317-1322. 


\section{Table $\mathbf{1}$ (on next page)}

Comparison of codon usage frequencies for ambiguous codons encoding $\mathrm{F}, \mathrm{I}, \mathrm{L}, \mathrm{M}$ and $\mathrm{V}$ in Escherichia coli and Streptomyces coelicolor.

Specific codons from two variant codons DTK and DTS are given, along with their codon usage frequency in the two organisms. For the amino acids $\mathrm{F}, \mathrm{I}$ and $\mathrm{V}$, there is a preference for codons with $\mathrm{T}$ in the third ("wobble") position in $E$. coli, and a preference for $\mathrm{C}$ in the wobble position for $\mathrm{S}$. coelicolor. This preference is reflected in the differences in scores for the ambiguous codons for the two organisms. 


\begin{tabular}{|c|c|c|c|c|}
\hline \multirow[t]{2}{*}{ Amino Acid } & \multirow[t]{2}{*}{ Codon } & \multirow[t]{2}{*}{ Ambiguous codon } & \multicolumn{2}{|c|}{ Codon usage frequency } \\
\hline & & & E. coli & S. coelicolor \\
\hline \multirow[t]{2}{*}{$\mathrm{F}$} & TTC & DTS & 0.36 & 0.97 \\
\hline & $\mathrm{TTT}$ & DTK & 0.64 & 0.03 \\
\hline \multirow[t]{2}{*}{ I } & $\mathrm{ATC}$ & DTS & 0.31 & 0.95 \\
\hline & $\mathrm{ATT}$ & DTK & 0.47 & 0.03 \\
\hline L & $\mathrm{TTG}$ & DTK and DTS & 0.13 & 0.03 \\
\hline M & ATG & DTK and DTS & 1.00 & 1.00 \\
\hline \multirow[t]{3}{*}{ V } & GTC & DTS & 0.19 & 0.58 \\
\hline & GTG & DTK and DTS & 0.29 & 0.36 \\
\hline & GTT & DTK & 0.32 & 0.02 \\
\hline
\end{tabular}




\section{Figure 1}

CodonGenie Design interface.

Users specify required amino acid combinations in the left-hand side panel. (Amino acids are grouped together in the interface in subsets of polar, non-polar, acidic and basic residues. In this example, the non-polar residues $A, F, G, I, L, M$ and $V$ have been selected.) Variant codons are listed in the Result panel, ordered by increasing number of Variants and decreasing codon Score (see Methods). The most specific codons are prioritised (e.g., the preferred codon in the above example, DBK, is [AGT][CGT][GT] and therefore encodes 18 DNA variants). Variant codons are shown in grey, with their encodings shown in green, orange and red for required amino acids, additional amino acids and stop codons, respectively. A given variant codon may encode an amino acid multiple times, and this is displayed in the output. For example, the preferred codon DBK encodes valine twice (with GTG and GTT) and these encodings and their codon usage frequencies may be visualised through a tooltip.

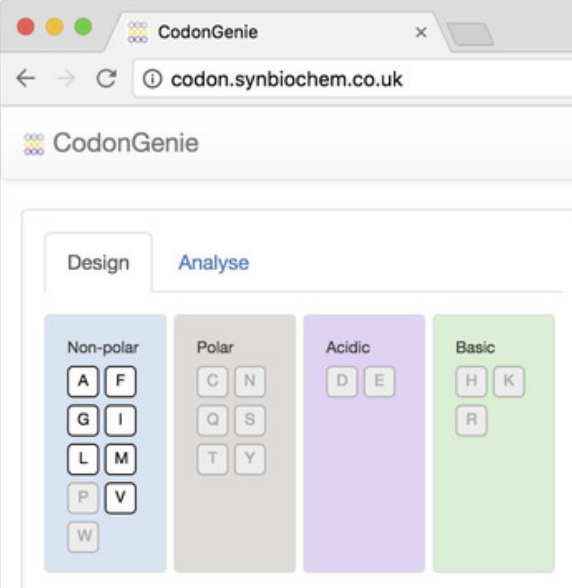

Organism:

Escherichia coli

\section{Result}

Codon Amino acids GTG (0.29), GT (0.32) Variants Score

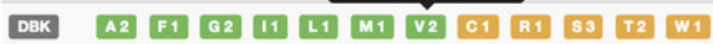

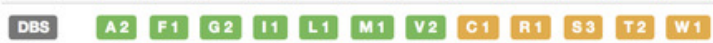

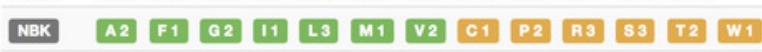

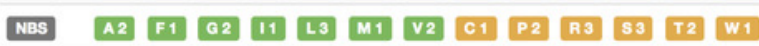

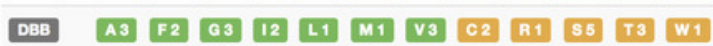

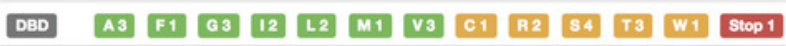

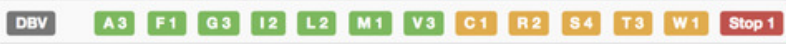

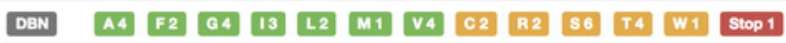

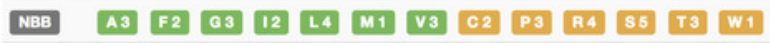

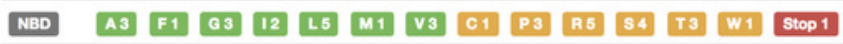

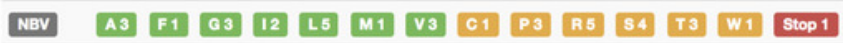

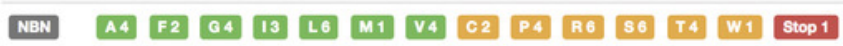

